Pacific

Journal of

Mathematics

ISOMETRIC EMBEDDING OF NEGATIVELY CURVED COMPLETE SURFACES IN LORENTZ-MINKOWSKI SPACE

BING-LONG Chen AND Le Yin 


\title{
ISOMETRIC EMBEDDING OF NEGATIVELY CURVED COMPLETE SURFACES IN LORENTZ-MINKOWSKI SPACE
}

\author{
BING-LONG CHEN AND LE YiN
}

\begin{abstract}
The Hilbert-Efimov theorem states that any complete surface with curvature bounded above by a negative constant cannot be isometrically embedded in $\mathbb{R}^{3}$. We demonstrate that any simply connected smooth complete surface with curvature bounded above by a negative constant admits a smooth isometric embedding into the Lorentz-Minkowski space $\mathbb{R}^{2,1}$.
\end{abstract}

\section{Introduction}

Weyl [1916] posed the problem whether every abstract compact smooth simply connected 2-dimensional Riemannian manifold with positive curvature can be isometrically embedded in $\mathbb{R}^{3}$. Weyl's problem was investigated by Weyl, Lewy, Alexandrov, and others, and finally resolved (in the smooth category) by Nirenberg [1953] and Pogorelov [1952] independently. The generalization to the nonnegative curvature case was done by Guan and Li [1994] and Hong and Zuily [1995], though only $C^{1,1}$ embedding was obtained.

For noncompact convex surfaces, the problem was solved by Olovjanisnikov in the 1940s in the weak sense (see the survey article [Hong 2001]), and in smooth category by Pogorelov [1973]. The result has been generalized to the nonnegatively curved case in [Hong 1997]. For local isometric embeddings, there were important breakthroughs by C.-S. Lin, Q. Han and J.-X. Hong [Lin 1985; 1986; Han et al. 2003; Han 2005; 2006] (see also the survey articles [Hong 2001; Yau 2000] and the book [Han and Hong 2006]).

The story is completely different for surfaces with negative curvature. The famous Hilbert-Efimov theorem [Hilbert 1901; Efimov 1964] asserts that any complete surface with curvature bounded above by a negative constant cannot be realized in $\mathbb{R}^{3}$. If the complete surface is negatively but not strongly negatively curved, Hong [1993] found a sufficient and almost sharp condition for the existence of an isometric embedding in $\mathbb{R}^{3}$.

The authors were partially supported by grants NSFC11025107 and NSFC11101289. MSC2010: primary 53C21; secondary 35J96.

Keywords: isometric embedding, Hilbert-Efimov theorem, Lorentz-Minkowski space. 
On the other hand, the hyperbolic plane $\mathbb{H}^{2}$ admits a canonical smooth isometric embedding in the 3-dimensional Lorentz-Minkowski space $\mathbb{R}^{2,1}$ as a unit imaginary sphere $x_{3}^{2}-\left(x_{1}^{2}+x_{2}^{2}\right)=1$. Here $\mathbb{R}^{2,1}$ denotes $\mathbb{R}^{3}$ equipped with the metric $d s^{2}=d x_{1}^{2}+d x_{2}^{2}-d x_{3}^{2}$. Instead of the Euclidean space $\mathbb{R}^{3}$, it is proved that the Lorentz-Minkowski space $\mathbb{R}^{2,1}$ is the appropriate ambient space for the isometric embedding of strongly negatively curved surfaces.

We remark that the problem of isometric embedding of Riemannian manifolds into Lorentzian manifolds has been investigated by many authors. Schlenker [2001] found a Hilbert-Efimov type theorem in the anti-de Sitter space. A celebrated theorem of Schoen and Yau [1981] states that a 3-dimensional complete asymptotic flat Riemannian manifold $\left(M^{3}, g, p\right)$ with dominant energy condition

$$
R-\left|p_{i j}\right|^{2}+\left(\operatorname{tr}_{g} p\right)^{2} \geq 2\left|\nabla_{j}\left(p^{i j}-\left(\operatorname{tr}_{g} p\right) g^{i j}\right)\right|_{g}
$$

and zero ADM mass, where $p_{i j}$ is a symmetric 2-tensor, admits an isometric embedding into $\mathbb{R}^{3,1}$ such that $p_{i j}$ is the second fundamental form. Delanoë [1988] and Guan [2007] constructed smooth isometric embeddings of a negatively curved compact 2-disc $\overline{\mathcal{D}}$ with smooth boundary $\partial \mathcal{D}$ into Lorentz-Minkowski space $\mathbb{R}^{2,1}$.

The purpose of this paper is to find global isometric embeddings for complete negatively curved surfaces into $\mathbb{R}^{2,1}$. The main result is the following:

Theorem 1.1. Let $(M, g)$ be a smooth 2-dimensional simply connected complete Riemannian manifold with curvature $K$ satisfying

$$
K \leq-C_{1}
$$

for some positive constant $C_{1}>0$. There exists a smooth isometric embedding $X: M \rightarrow \mathbb{R}^{2,1}$, and the spacelike submanifold $X(M)$ is a graph over $\mathbb{R}^{2} \subset \mathbb{R}^{2,1}$, say $\left(x_{1}, x_{2}, 0\right) \mapsto\left(x_{1}, x_{2}, Z\left(x_{1}, x_{2}\right)\right)$, satisfying

$$
\sqrt{x_{1}^{2}+x_{2}^{2}} \leq Z\left(x_{1}, x_{2}\right) \leq \sqrt{\frac{1}{C_{1}}+x_{1}^{2}+x_{2}^{2}} .
$$

Remark 1.2. It is likely that the solution of the isometric embedding problem is not unique (up to isometries of $\mathbb{R}^{2,1}$ ) if we drop the restriction (1-3). Actually, a remarkable fact (see [Guan et al. 2006]) is that there are many distinct isometric embeddings for the hyperbolic plane $\mathbb{H}^{2}$ into $\mathbb{R}^{2,1}$; some even have unbounded second fundamental forms and violate (1-3). In this sense, the natural isometric embedding provided by Theorem 1.1 is rather special. The construction and classification of all exotic embeddings deserve further study.

An interesting (but not direct) corollary of Theorem 1.1 is the following:

Theorem 1.3. Let $\left(M^{2}, g\right)$ be a compact 2-dimensional Riemannian manifold with negative Gaussian curvature. Then there is a smooth symmetric positive definite 
$(0,2)$-tensor $h_{i j}$, which is invariant under the isometry group of $\left(M^{2}, g\right)$, such that

$$
\begin{aligned}
R_{i j k l} & =-\left(h_{i k} h_{j l}-h_{i l} h_{j k}\right), \\
\nabla_{i} h_{j k} & =\nabla_{j} h_{i k},
\end{aligned}
$$

where $R_{i j k l}$ is the curvature tensor of the manifold.

The proof of Theorem 1.3 relies on a uniqueness theorem of isometric embeddings. Unfortunately, the uniqueness of the isometric embeddings in Theorem 1.1 has not been proven. Technically, the proof of the uniqueness theorem involves the estimates of the second fundamental form. Note that by Remark 1.2, it is possible that some exotic embeddings may violate these estimates. We remark that the boundedness of the second fundamental form is not sufficient to guarantee the uniqueness of the isometric embedding (see [Guan et al. 2006, Theorem 2.3]).

The proof of the main theorem is reduced to solving certain equations of MongeAmpère type

$$
\frac{\operatorname{det}\left(\nabla^{2} u+g\right)}{\operatorname{det}(g)}=-K_{g}\left(|\nabla u|^{2}+2 u\right)
$$

on the whole manifold $M$. The corresponding Dirichlet problems may be solved on a sequence of exhausting domains $\Omega_{l}$ with some particular boundary values. The problem amounts to deriving certain uniform a priori estimates for these solutions $u_{l}$. The bulk of the present paper is devoted to these estimates.

In Section 2, we sketch a proof of the main theorem and derive the zeroth- and first-order estimates. In Section 3, we derive the second- and higher-order estimates and prove Theorem 1.1. In Section 4, to prove Theorem 1.3, we derive some estimates of the second fundamental form (Theorem 4.1) and obtain a uniqueness theorem (Theorem 4.4) for isometric embeddings. Finally, in the appendix we supply an alternative, straightforward argument for the second-order derivative estimate.

\section{Zeroth- and first-order estimates}

Sketch of proof. Suppose $X: M \rightarrow \mathbb{R}^{2,1}$ is an isometric embedding. Then $X(M)$ is a spacelike submanifold and the Gauss-Codazzi-Weingarten equations read as follows:

$$
\begin{aligned}
\nabla_{i} \nabla_{j} X & =h_{i j} \vec{n}, \\
\nabla_{i} \vec{n} & =h_{i j} g^{j k} X_{k}, \\
R_{i j k l} & =-\left(h_{i k} h_{j l}-h_{i l} h_{j k}\right), \\
\nabla_{i} h_{j k} & =\nabla_{j} h_{i k},
\end{aligned}
$$


where $\vec{n}$ is the normal vector, $h_{i j}$ the second fundamental form, and $R_{i j k l}$ the curvature tensor. have

Let $u=-\frac{1}{2}\langle X, X\rangle$, where $\langle\cdot, \cdot\rangle$ is the Lorentz-Minkowski metric. By (2-1), we

$$
\begin{aligned}
\nabla_{i} u & =-\left\langle X, X_{i}\right\rangle, \\
\nabla_{i} \nabla_{j} u & =-h_{i j}\langle\vec{n}, X\rangle-g_{i j} .
\end{aligned}
$$

Since

$$
\begin{aligned}
\langle X, X\rangle & =\sum_{i, j=1}^{2} g^{i j}\left\langle X, X_{i}\right\rangle\left\langle X, X_{j}\right\rangle-\langle X, \vec{n}\rangle^{2} \\
& =|\nabla u|^{2}-\langle X, \vec{n}\rangle^{2}
\end{aligned}
$$

it follows that

$$
\langle X, \vec{n}\rangle^{2}=|\nabla u|^{2}+2 u .
$$

Combining (2-1), (2-2), and (2-3), we get

$$
\frac{\operatorname{det}\left(\nabla^{2} u+g\right)}{\operatorname{det}(g)}=-K_{g}\left(|\nabla u|^{2}+2 u\right) .
$$

Note that the equation (2-4) satisfied by the function $-\frac{1}{2}\langle X, X\rangle$ is an intrinsic equation on the manifold $(M, g)$.

Conversely, if we can find a bounded positive solution $u$ of (2-4) on $M$, we will show that this yields an isometric embedding $X:(M, g) \rightarrow \mathbb{R}^{2,1}$ such that $-\frac{1}{2}\langle X, X\rangle=u$. To construct this isometric embedding, we need to introduce the polar coordinates in the open future timelike cone

$$
\mathcal{I}^{+}=\left\{\left(x_{1}, x_{2}, x_{3}\right) \in \mathbb{R}^{2,1} \mid \sqrt{x_{1}^{2}+x_{2}^{2}}<x_{3}\right\} .
$$

In this polar coordinate system, the Lorentz-Minkowski metric takes the form

$$
-d r^{2}+r^{2} d s_{\square-1}^{2}
$$

where $r=\sqrt{x_{3}^{2}-x_{1}^{2}-x_{2}^{2}}$ and $d s_{\llbracket \rrbracket}^{2}$ is the hyperbolic metric $(K=-1)$ of the unit imaginary sphere $r=1$.

Proposition 2.1. For a positive $C^{2}$ function u on $M$, define a new metric

$$
\bar{g}=\frac{g+(d \sqrt{2 u})^{2}}{2 u}
$$


on $M$. The Gaussian curvature $K_{\bar{g}}$ of $\bar{g}$ is given by

$$
K_{\bar{g}}=-1+\frac{\frac{\operatorname{det}\left(\nabla^{2} u+g\right)}{\operatorname{det}(g)}+K_{g}\left(|\nabla u|^{2}+2 u\right)}{\left(1+\frac{|\nabla u|^{2}}{2 u}\right)^{2}} .
$$

Proof. The Gaussian curvature of the metric $g_{1} \triangleq g+(d \sqrt{2 u})^{2}$ can be computed by the formula

$$
K_{g_{1}}=\frac{1}{1+|\nabla \sqrt{2 u}|^{2}}\left(K_{g}+\frac{\operatorname{det}\left(\nabla^{2} \sqrt{2 u}\right)}{\operatorname{det}(g)\left(1+|\nabla \sqrt{2 u}|^{2}\right)}\right) .
$$

(see [Guan 2007; Hong 2001]). From

$$
g_{1}^{i j}=g^{i j}-\frac{u^{i} u^{j}}{2 u+|\nabla u|^{2}} \quad \text { and } \quad \Gamma_{1 i j}^{k}-\Gamma_{i j}^{k}=\frac{u^{k}}{2 u+|\nabla u|^{2}}\left(\nabla_{i j}^{2} u-\frac{u_{i} u_{j}}{2 u}\right)
$$

we may calculate $\Delta_{g_{1}} \log u$ by the formula

$$
\Delta_{g_{1}} \log u=g_{1}^{i j}\left(\nabla_{i j}^{2} \log u-\left(\Gamma_{1 i j}^{k}-\Gamma_{i j}^{k}\right)(\log u)_{k}\right),
$$

where $\nabla_{i j}^{2} \log u$ is the Hessian of $\log u$ with respect to the metric $g$. By the curvature formula of conformal transformation $\bar{g}=g_{1} / 2 u$, a long but straightforward computation gives us

$$
\frac{K_{\bar{g}}}{2 u}=K_{g_{1}}+\frac{1}{2} \Delta_{g_{1}} \log u=-\frac{1}{2 u}+\frac{1}{2 u} \frac{\frac{\operatorname{det}\left(\nabla^{2} u+g\right)}{\operatorname{det}(g)}+K_{g}\left(|\nabla u|^{2}+2 u\right)}{\left(1+\frac{|\nabla u|^{2}}{2 u}\right)^{2}} .
$$

Remark 2.2. If $u$ is a bounded positive smooth solution to (2-4), then the metric $\bar{g}$ in Proposition 2.1 is complete and has constant curvature -1 . Hence there exists an isometry $i:(M, \bar{g}) \rightarrow \mathbb{W}=\{r=1\}$ and we can construct an embedding $I:(M, g) \rightarrow \mathcal{I}^{+} \subset \mathbb{R}^{2,1}$ as $I(y) \triangleq(i(y), \sqrt{2 u(y)})$ in the polar coordinate system (2-5). It is clear that

$$
I^{*}\left(-d r^{2}+r^{2} d s_{\boxplus}^{2}\right)=-(d \sqrt{2 u})^{2}+2 u i^{*} d s_{\llbracket}^{2}=-(d \sqrt{2 u})^{2}+2 u \bar{g}=g,
$$

which shows that the map $I$ is the desired isometric embedding. The regularity of the embedding $I$ follows from the regularity of $u$.

Hence the proof of Theorem 1.1 may be reduced to solving equation (2-4). The next theorem gives the required solution.

Theorem 2.3. Under the assumptions of Theorem 1.1, equation (2-4) has a smooth bounded positive solution $u$ such that $0<u \leq 1 / 2 C_{1}$. 
The following strategy will be adapted to solve (2-4). We first solve it on a sequence of compact smooth exhausting domains $\Omega_{1} \Subset \Omega_{2} \Subset \cdots$. Let $u_{l}$ be the solution on $\Omega_{l}$. Fixing $x_{0} \in M$, we show that for any nonnegative integer $k \geq 0$, there exists a constant $D_{k}>0$ such that

$$
\sup _{\Omega_{l} \supset B\left(x_{0}, k+1\right)}\left|u_{l}\right|_{C^{k}\left(\bar{B}\left(x_{0}, k\right)\right)} \leq D_{k},
$$

where the norm $C^{k}\left(\bar{B}\left(x_{0}, k\right)\right)$ can be defined on some (indeed any) fixed finite coordinate covering of $\bar{B}\left(x_{0}, k\right)$. Once (2-8) has been obtained, we use the ArzelàAscoli theorem to extract a subsequence of $u_{l}$ such that the limit is a smooth solution of equation (2-4).

Indeed, we choose simply $\Omega_{l}=B\left(x_{0}, l\right)$ and consider the Dirichlet problem

$$
\left\{\begin{aligned}
\frac{\operatorname{det}\left(\nabla^{2} u+g\right)}{\operatorname{det}(g)} & =-K_{g}\left(|\nabla u|^{2}+2 u\right), \\
\left.u\right|_{\partial B\left(x_{0}, l\right)} & =\frac{1}{2 C_{2}(l)},
\end{aligned}\right.
$$

where $C_{2}(l)=\max _{x \in \bar{B}\left(x_{0}, l\right)}\left(-K_{g}(x)\right)$.

Clearly, (2-9) has a subsolution $u_{0} \equiv 1 / 2 C_{2}(l)$, i.e.,

$$
\frac{\operatorname{det}\left(\nabla^{2} u_{0}+g\right)}{\operatorname{det}(g)} \geq-K_{g}\left(\left|\nabla u_{0}\right|^{2}+2 u_{0}\right) .
$$

By continuity methods, this implies that (2-9) admits a smooth solution $u_{l}$ which satisfies $u_{l} \geq u_{0}$ and $\nabla^{2} u_{l}+g>0$ (see [Guan 1998]).

The main task of the subsequent sections is to derive a priori estimates for the solutions $u_{l}$ so that (2-8) holds. For convenience, we drop the subscript $l$ from $u_{l}$ and $\Omega_{l}$ in the process of the computations.

\section{Zeroth- and first-order estimates.}

Proposition 2.4. The solution $u$ of the Dirichlet problem (2-9) satisfies

$$
\frac{1}{2 C_{2}(l)} \leq u \leq \frac{1}{2 C_{1}} \text {. }
$$

Proof. By applying the maximum principle to $u$, we have

$$
\frac{1}{2 C_{2}(l)} \leq u \leq \max \left\{\sup _{\Omega}\left(-\frac{1}{2 K_{g}}\right), \frac{1}{2 C_{2}(l)}\right\} \leq \frac{1}{2 C_{1}} .
$$

Proposition 2.5 (first-order estimate). The gradient of the solution $u$ of (2-9) satisfies

$$
|\nabla u| \leq \frac{2}{\sqrt{C_{1}}}
$$


Proof. We choose

$$
\xi=\frac{1}{2 C_{2}(l)}+\frac{2}{\sqrt{C_{1}}}\left(l-d\left(x_{0}, \cdot\right)\right)
$$

as a barrier function. Clearly $\xi$ satisfies $\left.\xi\right|_{\partial \Omega}=1 / 2 C_{2}(l)$ and $|\nabla \xi| \leq 2 / \sqrt{C_{1}}$. By the standard Hessian comparison theorem

$$
\left.\operatorname{Hess}(d)\right|_{(\nabla d)^{\perp}} \geq \sqrt{C_{1}} \operatorname{coth}\left(d \sqrt{C_{1}}\right),
$$

we have

$$
\Delta \xi=-\frac{2}{\sqrt{C_{1}}} \Delta d \leq-2 .
$$

On the other hand, from $\nabla^{2} u+g>0$, we know that

$$
\Delta u+2>0 .
$$

Hence $\Delta(u-\xi)>0$ on $\Omega$. The maximum principle implies that $u \leq \xi$. Therefore we have

$$
\left.|\nabla u|\right|_{\partial \Omega} \leq|\nabla \xi| \leq \frac{2}{\sqrt{C_{1}}} .
$$

Now we consider the quantity $|\nabla u|^{2}+2 u$. The maximum $\max _{\bar{\Omega}}\left(|\nabla u|^{2}+2 u\right)$ is achieved either on the boundary or in the interior of the domain. In the former case, the maximum is bounded by $4 / C_{1}+1 / C_{2}(l)$ by (2-12). In the latter case, suppose the maximum is achieved at some point $\bar{x} \in \Omega$. Since

$$
0=\nabla_{i}\left(|\nabla u|^{2}+2 u\right)(\bar{x})=2\left(u_{i j}+g_{i j}\right) u_{j}(\bar{x})
$$

and $u_{i j}+g_{i j}>0$, it follows that $|\nabla u|(\bar{x})=0$, and therefore

$$
\max _{\bar{\Omega}}\left(|\nabla u|^{2}+2 u\right) \leq \max _{\bar{\Omega}}(2 u) \leq \frac{1}{C_{1}} .
$$

Combining both cases, we get

$$
\sup _{\bar{\Omega}}|\nabla u| \leq \max \left\{\frac{2}{\sqrt{C_{1}}}, \sqrt{\frac{1}{C_{1}}-\frac{1}{C_{2}(l)}}\right\}=\frac{2}{\sqrt{C_{1}}} .
$$

Propositions 2.4 and 2.5 state that the function $u$ and its gradient can be bounded from above by a constant independent of the domain $\Omega_{l}$. Before estimating the lower bound of $u$, we need to construct cutoff functions around points where the values of $u$ are not too large.

Lemma 2.6. Fix $\tilde{x} \in M$, and suppose there exist a real number $r_{0}>0$ and a solution $u$ of (2-9) defined on a domain $\Omega \supset B\left(\tilde{x}, r_{0}\right)$ satisfying

$$
u(\tilde{x})<\frac{r_{0}}{2 \sqrt{c_{2}} \operatorname{coth}\left(r_{0} \sqrt{c_{2}}\right)}, \quad \text { where } c_{2}=\max _{y \in \bar{B}\left(\tilde{x}, r_{0}\right)}\left(-K_{g}(y)\right) .
$$


Let $r_{2} \triangleq 2 \sqrt{c_{2}} \operatorname{coth}\left(r_{0} \sqrt{c_{2}}\right)$ be the denominator in (2-13). Then there are a domain $Q_{\tilde{x}} \subset B\left(\tilde{x}, r_{0}\right)$ containing $\tilde{x}$ and a function $\varphi^{\tilde{x}} \in C^{2}\left(\bar{Q}_{\tilde{x}}\right)$ such that

(i) $0 \leq \varphi^{\tilde{x}} \leq \frac{r_{0}}{r_{2}},\left.\quad \varphi^{\tilde{x}}\right|_{\partial Q_{\tilde{x}}}=0, \quad$ and

$$
\varphi^{\tilde{x}} \geq \frac{1}{2}\left(\frac{r_{0}}{r_{2}}-u(\tilde{x})\right) \quad \text { on } B\left(\tilde{x}, \frac{\sqrt{C_{1}}}{6}\left(\frac{r_{0}}{r_{2}}-u(\tilde{x})\right)\right)
$$

(ii) $\left|\nabla \varphi^{\tilde{x}}\right| \leq \frac{3}{\sqrt{C_{1}}}$;

(iii) $\nabla^{2} \varphi^{\tilde{x}} \geq-\left(\nabla^{2} u+g\right)$.

Proof. Set

$$
\xi=u+\frac{d^{2}(\tilde{x}, \cdot)}{r_{0} r_{2}}, \quad Q_{\tilde{x}}=\left\{\xi<\frac{r_{0}}{r_{2}}\right\}, \quad \varphi^{\tilde{x}}=\frac{r_{0}}{r_{2}}-\xi .
$$

Then $\varphi^{\tilde{x}}$ satisfies (ii) by Proposition 2.5. By (ii) and assumption (2-13), we know (i) holds. To check that $\varphi^{\tilde{x}}$ satisfies (iii), we use the Hessian comparison theorem

$$
\nabla^{2} d^{2}(\tilde{x}, \cdot) \leq 2 d(\tilde{x}, \cdot) \sqrt{c_{2}} \operatorname{coth}\left(d(\tilde{x}, \cdot) \sqrt{c_{2}}\right) g
$$

to conclude that

$$
\nabla^{2} \xi \leq \nabla^{2} u+g .
$$

Proposition 2.7 (lower bound for $u$ ). For any $\tilde{x} \in M, r_{0}>0$, assume the solution $u$ of (2-9) is defined on a domain $\Omega \supset B\left(\tilde{x}, r_{0}\right)$. Let $c_{2}$ and $r_{2}$ be defined as in Lemma 2.6. Then

$$
u(\tilde{x}) \geq \min \left\{\frac{r_{0}}{2 r_{2}}, \frac{C_{1} r_{0}^{2}}{9 r_{2}^{2}}, \frac{1}{32 c_{2}}\right\} .
$$

Proof. Assume

$$
u(\tilde{x})<\frac{r_{0}}{2 r_{2}} .
$$

Clearly the condition (2-13) holds for this $r_{0}$. Consider the quantity $u / \zeta$ around $\tilde{x}$, where $\zeta=\varphi^{\tilde{x}}$ is the cutoff function in Lemma 2.6. Suppose the minimum of $u / \zeta$ is achieved at some point $\bar{x} \in \operatorname{supp}(\zeta)$. At the point $\bar{x}$, we have

$$
\frac{\nabla u}{u}=\frac{\nabla \zeta}{\zeta} \quad \text { and } \quad 0 \leq \nabla^{2} \log \frac{u}{\zeta}=\frac{\nabla^{2} u}{u}-\frac{\nabla^{2} \zeta}{\zeta} .
$$

Diagonalize $u_{i j}=\lambda_{i} \delta_{i j}$ at $\bar{x}$ with an orthonormal basis. It follows from (2-17) that 


$$
\begin{aligned}
\sum \frac{\nabla_{i i} \zeta}{1+\lambda_{i}} & \leq \frac{\zeta}{u}\left(2-\frac{2}{\sqrt{\left(1+\lambda_{1}\right)\left(1+\lambda_{2}\right)}}\right) \\
& =\frac{2 \zeta}{u}\left(1-\frac{1}{\sqrt{\left(-K_{g}\right)\left(|\nabla \zeta|^{2} u^{2} / \zeta^{2}+2(u / \zeta) \zeta\right)}}\right) .
\end{aligned}
$$

Combining (2-18) and Lemma 2.6, we have

$$
-2 \leq \frac{2 \zeta}{u}\left(1-\frac{1}{\sqrt{\left(-K_{g}\right)\left(\frac{9}{C_{1}} \frac{u^{2}}{\zeta^{2}}+\frac{2 r_{0}}{r_{2}} \frac{u}{\zeta}\right)}}\right) .
$$

If the denominator in (2-19) is at most $\frac{1}{2}$, we get

$$
\frac{u}{\zeta} \geq 1
$$

On the other hand, if the denominator in (2-19) exceeds $\frac{1}{2}$, direct computation shows that

$$
\frac{u}{\zeta} \geq \min \left\{\frac{2 C_{1} r_{0}}{9 r_{2}}, \frac{r_{2}}{16 r_{0} c_{2}}\right\}
$$

Combining (2-20) and (2-21), we have

$$
u \geq \zeta \min \left\{1, \frac{2 C_{1} r_{0}}{9 r_{2}}, \frac{r_{2}}{16 r_{0} c_{2}}\right\} .
$$

Recalling (2-14) we immediately obtain (2-15).

Corollary 2.8. For any $r_{0}>0$, there is a constant $C$ depending only on $r_{0}$ and $C_{1}$ such that

$$
u(\tilde{x}) \geq \frac{C^{-1}}{\max _{\bar{B}\left(\tilde{x}, r_{0}\right)}\left(-K_{g}(x)\right)},
$$

for any solution $u$ to (2-9) defined on $\Omega \supset B\left(\tilde{x}, r_{0}\right)$.

\section{Second- and higher-order estimates}

In this section, we give a purely local second-order derivative estimate. This estimate could be done by Heinz-Lewy "characteristic" theory for Monge-Ampère equations in dimension 2. The reader is referred to the lecture notes [Schulz 1990] for detailed exposition. To state the result in [Schulz 1990], we consider the Monge-Ampère equation for a function $z=z(x, y)$ on a domain $\mathcal{D} \subset \mathbb{R}^{2}$ :

$$
\left(z_{x x}+C\right)\left(z_{y y}+A\right)-\left(z_{x y}-B\right)^{2}=K(x, y, z) D\left(x, y, z, z_{x}, z_{y}\right)>0,
$$

where $A, B, C, D$ are functions of $x, y, z, p, q$, and $p=z_{x}, q=z_{y}$. 
Assumption (i) $z \in C^{1,1}(\mathcal{D})$ and

$$
\left|z_{x}\right|+\left|z_{y}\right| \leq \mathcal{K}_{1} .
$$

Assumption (ii) $A, B, C \in C^{1}\left(\mathcal{D} \times \mathbb{R}^{3}\right), K \in C^{\mu}(\mathcal{D} \times \mathbb{R})$ for some $0<\mu<1$, $D \in C^{1}\left(\mathcal{D} \times \mathbb{R}^{3}\right)$, and

$$
\begin{gathered}
|A|+|B|+|C|+|D| \leq \mathcal{A}_{1}, \\
K, D \geq 1 / \mathcal{A}_{2}, \\
\left|\partial_{\mathcal{D} \times \mathbb{R}^{3}} A\right|+\cdots+\left|\partial_{\mathcal{D} \times \mathbb{R}^{3}} D\right| \leq \mathcal{A}_{3}, \\
|K|_{C^{\mu}(\mathcal{D} \times \mathbb{R})} \leq \mathcal{A}_{4} .
\end{gathered}
$$

Assumption (iii) The functions

$$
\begin{aligned}
& \phi_{1}(x, y)=A_{p}, \\
& \phi_{2}(x, y)=A_{q}+2 B_{p}, \\
& \phi_{3}(x, y)=C_{p}+2 B_{q}, \\
& \phi_{4}(x, y)=C_{q}
\end{aligned}
$$

are Lipschitz continuous with

$$
\left[\phi_{1}\right]_{0,1}^{\mathcal{D}}+\cdots+\left[\phi_{4}\right]_{0,1}^{\mathcal{D}} \leq \mathcal{A}_{5} .
$$

Theorem 3.1 [Schulz 1990, Theorem 9.4.1]. Suppose $z \in C^{1,1}(\mathcal{D})$ is a solution of (3-1) such that the above Assumptions (i), (ii), and (iii) hold with the constants $\mathcal{K}_{1}, \mathcal{A}_{1}, \ldots, \mathcal{A}_{5}$. Then $z \in C_{\text {loc }}^{2, \mu}(\mathcal{D})$, and for any $\mathcal{D}^{\prime} \Subset \mathcal{D}$ there is an interior estimate

$$
\left\|\partial^{2} z\right\|_{C^{\mu}\left(\mathcal{D}^{\prime}\right)} \leq C\left(\mu, \mathcal{K}_{1}, \mathcal{A}_{1} \cdots \mathcal{A}_{5}, \operatorname{dist}\left(\mathcal{D}^{\prime}, \partial \mathcal{D}\right)\right) .
$$

For any $\tilde{x} \in M$, to invoke the result in [Schulz 1990], we fix a local coordinate system $(x, y) \in \mathcal{D}$ in $M$ around $\tilde{x}$. Take $z(x, y)$ to be a solution $u(x, y)$ of equation (2-9) defined on $\Omega \supset \mathcal{D}$. Then we find

$$
\begin{aligned}
A & =g_{22}-\Gamma_{22}^{k} p_{k}, \\
B & =-g_{12}+\Gamma_{12}^{k} p_{k}, \\
C & =g_{11}+\Gamma_{11}^{k} p_{k}, \\
D & =g^{k l} p_{k} p_{l}+2 z, \\
K(x, y, z) & =-K_{g}(x, y) \operatorname{det}\left(g_{i j}\right),
\end{aligned}
$$

where $p_{1}=p, p_{2}=q$.

Note that by Propositions 2.4, 2.5, and 2.7, we have estimated the upper bound of $u, \nabla u$, and the lower bound of $u$ in the coordinate system $\mathcal{D}$. This gives rise to a 
control of the constants $\mathcal{K}_{1}, \mathcal{A}_{1}, \ldots, \mathcal{A}_{5}$ in terms of the geometry of $(\mathcal{D}, g)$. From Theorem 3.1, we have immediately

Proposition 3.2. For any nonnegative integer $k \geq 0$, there exists a constant $D_{k}>0$ such that

$$
\sup _{\Omega_{l} \supset B\left(x_{0}, k+1\right)}\left|u_{l}\right|_{C^{2, \mu}\left(\bar{B}\left(x_{0}, k+\frac{1}{2}\right)\right)} \leq D_{k},
$$

where the norm $C^{2, \mu}\left(\bar{B}\left(x_{0}, k+\frac{1}{2}\right)\right)$ can be defined on some (and any) fixed finite coordinate covering of $\bar{B}\left(x_{0}, k+\frac{1}{2}\right)$.

We proceed to consider the third- and higher-order estimates (2-8). This may be done by the standard Schauder estimate for elliptic equations.

Proposition 3.3. For any nonnegative integer $k \geq 0$, there exists a constant $D_{k}>0$ such that

$$
\sup _{\Omega_{l} \supset B\left(x_{0}, k+1\right)}\left|u_{l}\right|_{C^{k}\left(\bar{B}\left(x_{0}, k\right)\right)} \leq D_{k},
$$

where the norm $C^{k}\left(\bar{B}\left(x_{0}, k\right)\right)$ can be defined on some (indeed any) fixed finite coordinate covering of $\bar{B}\left(x_{0}, k\right)$.

Proof. By (2-9), we see that $\nabla_{i} u$ satisfies an equation of the type

$$
\hat{g}^{j m} v_{j m}=f(x, v, \nabla v),
$$

where $\hat{g}=\nabla^{2} u+g$. By the previous second-order estimate, we know (3-13) is uniformly elliptic on $B\left(x_{0}, k+\frac{1}{2}\right)$ and the $C^{\mu}$ norm of $\hat{g}$ and $f$ are uniformly bounded (independently of $l$ ). The result follows from the standard interior Schauder estimate and a bootstrap argument.

Proof of Theorem 2.3. By Proposition 3.3 and the Arzelà-Ascoli theorem, we may extract a $C_{\text {loc }}^{\infty}$ convergent subsequence of $u_{l}$. The limit is the desired solution.

Theorem 1.1 follows from Theorem 2.3 (see Remark 2.2).

\section{Estimating the second fundamental form}

In this section, we refine the result in Theorem 1.1. We prove that under an additional "smoothness" assumption on the Gaussian curvature, a particular embedding with controlled second fundamental form will be obtained. More precisely, we have

Theorem 4.1. Let $(M, g)$ be a smooth 2-dimensional simply connected complete Riemannian manifold whose Gaussian curvature satisfies

$$
-C_{2} \leq K \leq-C_{1}
$$


and

$$
\sup _{d(x, y) \leq 1} \frac{|K(x)-K(y)|}{d(x, y)^{\mu}} \leq C_{\mu}
$$

for some positive constants $C_{2} \geq C_{1}>0,1>\mu>0, C_{\mu}>0$.

Then there exists a smooth isometric embedding $X: M \rightarrow \mathbb{R}^{2,1}$ such that the spacelike submanifold $X(M)$ is a graph over $\mathbb{R}^{2} \subset \mathbb{R}^{2,1}:\left(x_{1}, x_{2}, 0\right) \rightarrow\left(x_{1}, x_{2}, Z\left(x_{1}, x_{2}\right)\right)$ satisfying these conditions:

$$
\sqrt{\frac{1}{C_{2}}+x_{1}^{2}+x_{2}^{2}} \leq Z\left(x_{1}, x_{2}\right) \leq \sqrt{\frac{1}{C_{1}}+x_{1}^{2}+x_{2}^{2}} .
$$

(ii) $|A| \leq C$, where $A$ is the second fundamental form of the submanifold $X(M)$, and the constant $C$ only depends on $C_{1}, C_{2}$, and $C_{\mu}$.

The proof of Theorem 4.1 is based on Proposition 2.4 and Theorem 3.1 and the following result.

Proposition 4.2. Under the assumptions (4-1) and (4-2) of Theorem 4.1, there exists $R>0$ such that $M$ admits a covering of isothermal coordinate charts $\left\{\left(U_{i},\left(u^{1}, u^{2}\right)\right)\right\}$, where with $U_{i}=\left\{\left(u^{1}\right)^{2}+\left(u^{2}\right)^{2}<R^{2}\right\}$, with these properties:

(i) For any $y_{0} \in M$, there is $U_{i_{0}}$ with $y_{0} \in\left\{\left(u^{1}\right)^{2}+\left(u^{2}\right)^{2}<R^{2} / 4\right\} \subset U_{i_{0}}$.

(ii) In each $U_{i}$, the metric $g$ of $M$ takes the form $g=\psi\left(\left(d u^{1}\right)^{2}+\left(d u^{2}\right)^{2}\right)$, with

$$
c^{-1} \leq \psi \leq c \text { and }|\psi|_{C^{2, \mu}\left(U_{i}\right)} \leq c_{\mu},
$$

for constants $c$ and $c_{\mu}$ independent of $i$. If additionally (4-6) is satisfied, we have

$$
|\psi|_{C^{l+1, \alpha}\left(U_{i}\right)} \leq c_{l, \alpha} \quad \text { for any } \alpha \in(0,1),
$$

where the $c_{l, \alpha}$ are constants independent of $i$.

Proof of Theorem 4.1. By Proposition 2.4 and Theorem 1.1, we know there exists a smooth isometric embedding $X: M \rightarrow \mathbb{R}^{2,1}$ such that $u=-\frac{1}{2}\langle X, X\rangle$ satisfying $1 / 2 C_{2} \leq u \leq 1 / 2 C_{1}$. Let $R$ be the constant provided in Proposition 4.2. Let the coordinates $(x, y)$ in equation (3-1) be the isothermal coordinates $\left(u^{1}, u^{2}\right)$ in Proposition 4.2, $z(x, y)=u(x, y)$, and $\mathcal{D}=\left\{x^{2}+y^{2}<R^{2} / 4\right\}$. In these coordinates, (3-10) becomes

$$
\begin{aligned}
A & =\psi-\Gamma_{22}^{k} p_{k}, \\
B & =\Gamma_{12}^{k} p_{k}, \\
C & =\psi+\Gamma_{11}^{k} p_{k}, \\
D & =\psi^{-1}\left(p_{1}^{2}+p_{2}^{2}\right)+2 z, \\
K(x, y, z) & =-K_{g}(x, y) \psi^{2} .
\end{aligned}
$$


Estimate (4-3) and Proposition 2.5 imply that there is a constant $C$ depending only on $C_{1}, C_{2}, C_{\mu}$ such that the constants in equations (3-2)-(3-8) can be bounded by $C$,

$$
\mathcal{K}_{1}, \mathcal{A}_{1}, \ldots, \mathcal{A}_{5} \leq C
$$

Theorem 3.1 implies $\left|\partial_{i j} u\right|_{C^{\mu}(B(0, R / 4))} \leq C$. Combining this with (4-3) in particular gives $\left|h_{i j}\right| \leq C$. This proves (ii) in Theorem 4.1.

Remark 4.3. If the curvature covariant derivatives up to order $l$ are assumed to be bounded in Theorem 4.1, i.e.,

$$
\sum_{p=0}^{l}\left|\nabla^{p} K\right| \leq \bar{C}_{l}
$$

for some $l \geq 1$, then the covariant derivatives of the second fundamental form of $X(M)$ up to order $l-1$ are also bounded,

$$
\sup _{x \in X(M)} \sum_{p=0}^{l-1}\left|\nabla^{p} A\right|(x) \leq C
$$

for some $C$ depending only on $\bar{C}_{l}$.

Actually, if (4-6) is assumed, notice that (4-4) holds. Then (4-7) follows by the same argument as in Proposition 3.3.

An important application of Theorem 4.1 is to give a uniqueness theorem.

Theorem 4.4. Under the assumptions of Theorem 4.1, let $X$ be the isometric embedding constructed in Theorem 4.1.

(i) Let $\widetilde{X}$ be another isometric embedding of $(M, g)$ into $\mathbb{R}^{2,1}$ such that $\widetilde{X}(M)$ is represented as a graph

$$
\sqrt{y_{1}^{2}+y_{2}^{2}} \leq \tilde{Z}\left(y_{1}, y_{2}\right) \leq \sqrt{\frac{1}{C}+y_{1}^{2}+y_{2}^{2}}
$$

in some Lorentz-Minkowski coordinate system $\left\{y_{1}, y_{2}, y_{3}\right\}$. Then there is an isometry $\iota \in \operatorname{Iso}\left(\mathbb{R}^{2,1}\right)$ such that $\tilde{X}=\iota \circ X$.

(ii) There is an injective homomorphism $\rho: \operatorname{Iso}(M, g) \rightarrow \operatorname{Iso}(\mathbb{M}) \subset \operatorname{Iso}\left(\mathbb{R}^{2,1}\right)$ such that

$$
X \circ \gamma=\rho(\gamma) \circ X
$$

for any $\gamma \in \operatorname{Iso}(M, g)$, where $\operatorname{Iso}(M, g)$, Iso $(\mathbb{H})$, and $\operatorname{Iso}\left(\mathbb{R}^{2,1}\right)$ are the groups of isometries of $M$, the unit imaginary sphere in $\mathbb{R}^{2,1}$, and $\mathbb{R}^{2,1}$ respectively. 
Proof. After an isometry $\tilde{\iota}$ of $\mathbb{R}^{2,1}, \tilde{\iota} \circ \tilde{X}(M)$ can be pinched between the light cone and a hyperboloid associated to $X$, and we can define $\tilde{u}=-\frac{1}{2}\langle\tilde{\iota} \circ \tilde{X}, \tilde{\iota} \circ \tilde{X}\rangle$, which satisfies $0<\tilde{u} \leq C$.

Using the polar coordinates in Remark 2.2 , we know that $\tilde{\iota} \circ \tilde{X}$ is determined by $\tilde{u}$ and an isometry

$$
\tilde{i}:\left(M, \frac{g+(d \sqrt{2 \tilde{u}})^{2}}{2 \tilde{u}}\right) \rightarrow \mathbb{} .
$$

To show that $\tilde{\iota} \circ \tilde{X}$ is congruent to $X$, it suffices to show that $u=\tilde{u}$. Indeed, once we have $u=\tilde{u}$, it follows that $\tilde{\iota} \circ \tilde{X}=\sigma \circ X$, where $\sigma=\tilde{i} i^{-1} \in \operatorname{Iso}(\mathbb{M}) \subset \operatorname{Iso}\left(\mathbb{R}^{2,1}\right)$. Then $\tilde{X}=\iota \circ X$, where $\iota=\tilde{\iota}^{-1} \circ \sigma$.

We need some a priori estimates of $\tilde{u}$ up to second order. To this end, we use the powerful tool of the maximum principle in [Cheng and Yau 1980]. Since the curvature is assumed to be bounded, for any $C^{2}$ function $F$ bounded from above, there is a sequence of $x_{k} \in M$ and $\varepsilon_{k} \rightarrow 0$ such that

$$
\begin{aligned}
\sup _{M} F-F\left(x_{k}\right) & \leq \varepsilon_{k}, \\
|\nabla F|\left(x_{k}\right) & \leq \varepsilon_{k}, \\
\nabla^{2} F\left(x_{k}\right) & \leq \varepsilon_{k} g .
\end{aligned}
$$

Note that $\tilde{u}$ satisfies (2-4). Applying the above maximum principle to $\tilde{u}$ and $-\tilde{u}$, we immediately get

$$
\frac{1}{2 C_{2}} \leq \tilde{u} \leq \frac{1}{2 C_{1}} .
$$

We claim that the gradient of $\tilde{u}$ is also bounded, and more precisely, it satisfies

$$
|\nabla \tilde{u}| \leq \frac{1}{\sqrt{C_{1}}}
$$

Indeed, for any $\tilde{x} \in M$, let $\gamma$ be a geodesic of unit speed such that $\gamma(0)=\tilde{x}$. We would like to control $\left|\frac{d}{d t}(\tilde{u} \circ \gamma)(0)\right|$. By the convexity of the function $\tilde{u}+\frac{1}{2} d^{2}(\tilde{x}, \ldots)$, we know

$$
t\left|\frac{d}{d t}(\tilde{u} \circ \gamma)(0)\right| \leq \max \{\tilde{u}(\gamma(t))-\tilde{u}(\gamma(0)), \tilde{u}(\gamma(-t))-\tilde{u}(\gamma(0))\}+\frac{t^{2}}{2} .
$$

It follows that $\left|\frac{d}{d t}(\tilde{u} \circ \gamma)(0)\right| \leq 1 / \sqrt{C_{1}}$ by taking $t=1 / \sqrt{C_{1}}$. This implies $|\nabla \tilde{u}|(\tilde{x}) \leq$ $1 / \sqrt{C_{1}}$, and the claim is proved.

Combining the gradient estimate of $\tilde{u}$ with the proof of Proposition 3.2, we know that $\left|\nabla^{2} \tilde{u}\right|$ is bounded.

Summarizing the above estimates, it follows that there is $C>0$ such that

$$
1 / C \leq u_{t} \leq C, \quad\left|\nabla u_{t}\right| \leq C, \quad \nabla^{2} u_{t}+g \geq C^{-1} g,
$$

where $u_{t}=u+t(\tilde{u}-u), t \in[0,1]$. 
Note that $u$ and $\tilde{u}$ satisfy the same equation (2-4). This implies

$$
\int_{0}^{1}\left(g+\nabla^{2} u_{t}\right)^{i j} d t \nabla_{i j}^{2}(\tilde{u}-u)=\int_{0}^{1} \frac{2\left\langle\nabla u_{t}, \nabla(\tilde{u}-u)\right\rangle+2(\tilde{u}-u)}{\left|\nabla u_{t}\right|^{2}+2 u_{t}} d t
$$

Let $F=\tilde{u}-u$ in (4-10). Combining (4-10) with (4-11) and (4-12), we have

$$
C \varepsilon_{k} \geq \sup _{M}(\tilde{u}-u)
$$

This gives $u \geq \tilde{u}$. Similarly, we have $u \leq \tilde{u}$. Hence $u=\tilde{u}$.

To prove (ii), one can show $u \circ \gamma=u$ for any $\gamma \in \operatorname{Iso}(M, g)$ by Cheng and Yau's maximum principle (4-10). This implies $\operatorname{Iso}(M, g) \subset \operatorname{Iso}(M, \bar{g})$, where

$$
\bar{g}=\frac{g+(d \sqrt{2 u})^{2}}{2 u} .
$$

The desired injective homomorphism $\rho: \operatorname{Iso}(M, g) \rightarrow \operatorname{Iso}(\mathbb{M})$ is given by

$$
\rho(\gamma)=i \circ \gamma \circ i^{-1}
$$

Proof of Theorem 1.3. Let $(\tilde{M}, \tilde{g})$ be the universal cover of $(M, g)$ with the induced metric. Then the curvature of $\tilde{g}$ is pinched between two negative constants, and all covariant derivatives of the curvature are bounded. By Theorem 4.4, there is an isometric embedding $X:(\tilde{M}, \tilde{g}) \rightarrow \mathbb{R}^{2,1}$ and an injective homomorphism $\rho: \operatorname{Iso}(\tilde{M}, \tilde{g}) \rightarrow \operatorname{Iso}\left(\mathbb{R}^{2,1}\right)$ such that (4-9) holds. Let $\tilde{h}_{i j}$ be the second fundamental form of $X$. Note that the deck transformation group $\Gamma$ of $\tilde{M}$ over $M$ is contained in $\operatorname{Iso}(\tilde{M}, \tilde{g})$. Combining (4-9), we know $\tilde{h}_{i j}$ is invariant under $\Gamma$. This implies $\tilde{h}_{i j}$ descends to a tensor $h_{i j}$ on $M$. This completes the proof of Theorem 1.3.

\section{Appendix}

The purpose of this appendix is to give an alternative method for the second-order estimate. The argument we present here is classical, straightforward, and may be generalized to higher dimensions (see [Guan and Li 1996]). The price to be paid is that this method requires some geometry of the background manifold. It works well on those points where the values of a solution $u$ of (2-9) are not too large in comparison to the local geometry.

Proposition 5.1. There exists $C>0$, depending only on $C_{1}$, satisfying the following property. Fixing $\tilde{x} \in M$, suppose there exist a real number $r_{0}>0$ and a solution $u$ of (2-9) defined on a domain $\Omega_{l} \supset B\left(\tilde{x}, r_{0}\right)$ such that

$$
u(\tilde{x})<\frac{r_{0}}{2 \sqrt{c_{2}} \operatorname{coth}\left(r_{0} \sqrt{c_{2}}\right)}, \quad \text { where } c_{2}=\max _{y \in \bar{B}\left(\tilde{x}, r_{0}\right)}\left(-K_{g}(y)\right) .
$$


Let $r_{2} \triangleq 2 \sqrt{c_{2}} \operatorname{coth}\left(r_{0} \sqrt{c_{2}}\right)$. Then

$$
\left(g+\nabla^{2} u\right)(x) \leq \frac{e^{C c_{2}^{\prime}}}{r_{0} / r_{2}-u(\tilde{x})}\left(1+\sqrt{c_{4}} \frac{r_{0}}{\sqrt{c_{2}}}+c_{2}^{\prime}\left(1+\frac{r_{0}}{\sqrt{c_{2}}}+c_{3} \frac{r_{0}}{\sqrt{c_{2}}}\right)\right)
$$

on $B\left(\tilde{x}, \frac{1}{6} \sqrt{C_{1}}\left(r_{0} / r_{2}-u(\tilde{x})\right)\right)$, where

$$
\begin{aligned}
& c_{2}^{\prime}=\max _{x \in \bar{B}\left(\tilde{x}, r_{0}+1\right)}\left(-K_{g}(x)\right), \\
& c_{3}=\max _{x \in \bar{B}\left(\tilde{x}, r_{0}\right)}\left|\nabla \log \left(-K_{g}(x)\right)\right|, \\
& c_{4}=\max _{x \in \bar{B}\left(\tilde{x}, r_{0}\right)}\left|\nabla^{2} \log \left(-K_{g}(x)\right)\right| .
\end{aligned}
$$

Note that by Proposition 2.4, condition (5-1) can be justified at each $\tilde{x}$ (for suitable $r_{0}$ ) when the curvature $K$ satisfies

$$
-C_{2}^{2}\left(d\left(x, x_{0}\right)+C_{3}\right)^{2} \leq K(x) \leq-C_{1}
$$

for some $x_{0} \in M$ and positive constants $0<C_{2}<C_{1}<C_{3}$.

Proof of Proposition 5.1. Consider an auxiliary function $S T M \rightarrow \mathbb{R}$ on the unit tangent bundle of $M$, given by

$$
(x, \gamma) \mapsto \eta(x)\left(1+\nabla_{\gamma \gamma} u\right) e^{\frac{a}{2}\left(|\nabla u|^{2}+2 u\right)(x)},
$$

where $x \in M, \gamma \in T_{x} M,|\gamma|=1, \eta$ is a cutoff function on $M$, and $a \geq 1$ is a constant to be specified later. Suppose the maximum

$$
\max _{(x, \gamma) \in S T M} \eta\left(1+\nabla_{\gamma \gamma} u\right) e^{\frac{a}{2}\left(|\nabla u|^{2}+2 u\right)}
$$

is achieved at $\bar{x} \in \operatorname{supp}(\eta)$ for some $\gamma \in T_{\bar{x}} M$ with $|\gamma|=1$. Diagonalize $u_{i j}=\lambda_{i} \delta_{i j}$ at $\bar{x}$ with the orthonormal eigenvectors $e_{i}$. Let $e_{1}=\gamma$. By parallel transport of each $e_{i}$ along radial geodesics, we obtain a field of orthonormal frame $\left\{e_{i}\right\}$ near $\bar{x}$. The function

$$
w=\eta\left(1+\nabla_{e_{1}, e_{1}} u\right) e^{\frac{a}{2}\left(|\nabla u|^{2}+2 u\right)}
$$

defined near $\bar{x}$ achieves its maximum at $\bar{x}$. In the following, we use $C$ to denote various big constants depending only on $C_{1}$.

At the point $\bar{x}$, we have

$$
0=\nabla_{i} \log w=\frac{\nabla_{i 11} u}{1+\lambda_{1}}+a\left(1+\lambda_{i}\right) u_{i}+\frac{\nabla_{i} \eta}{\eta}
$$


and

$$
\begin{aligned}
0 & \geq \nabla_{i j} \log w \\
& =\frac{\nabla_{i j 11} u}{1+\lambda_{1}}-\frac{\nabla_{i 11} u \nabla_{j 11} u}{\left(1+\lambda_{1}\right)^{2}}+a\left(u_{k} \nabla_{i j k} u+\left(\lambda_{i}+\lambda_{i}^{2}\right) \delta_{i j}\right)+\frac{\nabla_{i j} \eta}{\eta}-\frac{\nabla_{i} \eta \nabla_{j} \eta}{\eta^{2}}
\end{aligned}
$$

Let $\left.f=f(x, z, p) \triangleq \log (-K)+\log \left(|\nabla u|^{2}+2 u\right)\right)$, where $z=u, p=\nabla u$. Differentiating equation (2-9), we get

$$
f_{11}=\frac{\nabla_{11 i i} u}{1+\lambda_{i}}-\frac{\left(\nabla_{1 i j} u\right)^{2}}{\left(1+\lambda_{i}\right)\left(1+\lambda_{j}\right)} \quad \text { and } \quad \nabla_{k} f=\frac{\nabla_{k i i} u}{1+\lambda_{i}} .
$$

Combining (5-7), (5-8), and the Ricci formula, we have

$$
\begin{aligned}
\left(1+\lambda_{1}\right)\left(-\frac{\nabla_{i i} \eta}{\left(1+\lambda_{i}\right) \eta}+\frac{\left|\nabla_{i} \eta\right|^{2}}{\left(1+\lambda_{i}\right) \eta^{2}}\right) & \\
& +\frac{1}{1+\lambda_{i}}\left(\frac{\left(\nabla_{i 11} u\right)^{2}}{1+\lambda_{1}}-a\left(R_{i j i p} u_{p} u_{j}+\lambda_{i}+\lambda_{i}^{2}\right)\left(1+\lambda_{1}\right)\right. \\
& \left.-\left(\nabla_{i} R_{i 11 p}+\nabla_{1} R_{i 1 i p}\right) u_{p}-2 R_{1 i 1 i}\left(\lambda_{1}-\lambda_{i}\right)\right)-\frac{\left(\nabla_{1 i j} u\right)^{2}}{\left(1+\lambda_{i}\right)\left(1+\lambda_{j}\right)} \\
& \geq f_{11}+a u_{j} \nabla_{j} f\left(1+\lambda_{1}\right) .
\end{aligned}
$$

By direct computations, we have (at $\bar{x}$ )

(5-10) $\quad f_{11}+a u_{k} \nabla_{k} f\left(1+\lambda_{1}\right) \geq$

$$
\begin{aligned}
\frac{2}{|\nabla u|^{2}+2 u} & \left\langle\nabla u,-a \nabla u-\frac{\nabla \eta}{\eta}\right\rangle\left(1+\lambda_{1}\right) \\
& +\log (-K)_{11}+a\langle\nabla \log (-K), \nabla u\rangle\left(1+\lambda_{1}\right) \\
& +\frac{a\left(1+\lambda_{1}\right)|\nabla u|^{2}}{|\nabla u|^{2}+2 u}-\frac{8\left(\left|\lambda_{1}\right|+1\right)^{2}}{|\nabla u|^{2}+2 u}+2 \frac{R_{1 j 1 l} u_{j} u_{l}}{|\nabla u|^{2}+2 u} .
\end{aligned}
$$

By (5-6), we have

$$
\begin{aligned}
& \sum_{i} \frac{\left|\nabla_{i} \eta\right|^{2}}{\left(1+\lambda_{i}\right) \eta^{2}}\left(1+\lambda_{1}\right) \\
& =\frac{\left|\nabla_{1} \eta\right|^{2}}{\eta^{2}}+\sum_{i \geq 2}\left(\frac{\left|\nabla_{i 11} u\right|^{2}}{\left(1+\lambda_{1}\right)\left(1+\lambda_{i}\right)}\right. \\
& \left.\quad-2 a\left(1+\lambda_{1}\right) \nabla_{i} u \frac{\nabla_{i} \eta}{\eta}-a^{2}\left(1+\lambda_{1}\right)\left(1+\lambda_{i}\right) u_{i}^{2}\right) .
\end{aligned}
$$


Note that

$$
\begin{array}{r}
\sum_{i, j} \frac{\left(\nabla_{1 i j} u\right)^{2}}{\left(1+\lambda_{i}\right)\left(1+\lambda_{j}\right)}-\sum_{i} \frac{\left(\nabla_{i 11} u\right)^{2}}{\left(1+\lambda_{1}\right)\left(1+\lambda_{i}\right)}-\sum_{i \geq 2} \frac{\left|\nabla_{i 11} u\right|^{2}}{\left(1+\lambda_{1}\right)\left(1+\lambda_{i}\right)} \\
\geq-4 \frac{|\nabla u|}{|\nabla u|^{2}+2 u} \frac{|\nabla \eta|}{\eta}\left(1+\lambda_{1}\right),
\end{array}
$$

$$
\begin{aligned}
\sum_{i}-\frac{1}{1+\lambda_{i}}\left(R_{i j i p} u_{p} u_{j}+\lambda_{i}+\lambda_{i}^{2}\right) & \left(1+\lambda_{1}\right) \\
& \leq-\frac{2 u}{|\nabla u|^{2}+2 u}\left(1+\lambda_{1}\right)^{2}+2\left(1+\lambda_{1}\right),
\end{aligned}
$$

$$
\begin{aligned}
\frac{1}{1+\lambda_{i}} & \left(-\left(\nabla_{i} R_{i 11 p}+\nabla_{1} R_{i 1 i p}\right) u_{p}-2 R_{1 i 1 i}\left(\lambda_{1}-\lambda_{i}\right)\right) \\
& \leq \frac{2|\nabla u|}{|\nabla u|^{2}+2 u}|\nabla \log (-K)|\left(1+\lambda_{1}\right)+\frac{2}{|\nabla u|^{2}+2 u}\left(1+\lambda_{1}\right)^{2}+2 K
\end{aligned}
$$

Multiplying both sides of (5-9) by $\eta^{2}$ and combining (5-10)-(5-14), we get

$$
L_{1}\left(1+\lambda_{1}\right)^{2} \eta^{2}-L_{2}\left(1+\lambda_{1}\right) \eta-L_{3} \leq \eta\left(1+\lambda_{1}\right) \sum_{i \geq 1} \frac{-\nabla_{i i} \eta}{1+\lambda_{i}},
$$

where

$$
\begin{aligned}
& L_{1}=a \frac{2 u}{|\nabla u|^{2}+2 u}-\frac{10}{|\nabla u|^{2}+2 u}, \\
& L_{2}=\left(6 \frac{|\nabla u|}{|\nabla u|^{2}+2 u}+2 a|\nabla u|\right)|\nabla \eta|+2 a \eta+\frac{|\nabla u|^{2}}{|\nabla u|^{2}+2 u} a \eta \\
& +a|\nabla \log (-K)||\nabla u| \eta+\frac{2|\nabla u|}{|\nabla u|^{2}+2 u}|\nabla \log (-K)| \eta, \\
& L_{3}=|\nabla \eta|^{2}+\eta^{2}\left|\nabla^{2} \log (-K)\right|+2 K \frac{\left|\nabla_{1} u\right|^{2}+2 u}{|\nabla u|^{2}+2 u} \eta^{2} \text {. }
\end{aligned}
$$

Note that by (5-1), Lemma 2.6 is applicable. Choose the cutoff function $\eta$ in (5-5) to be $\varphi^{\tilde{x}}$ in Lemma 2.6 , and consider the maximum of the quantity $w$ on $Q_{\tilde{x}}$. From Lemma 2.6 (iii), we have

$$
\eta\left(1+\lambda_{1}\right) \sum_{i \geq 1} \frac{-\nabla_{i i} \eta}{1+\lambda_{i}} \leq 2\left(1+\lambda_{1}\right) \eta .
$$

Since $u(\bar{x}) \geq C^{-1} c_{2}^{\prime-1}$ by Corollary 2.8, choosing $a=10 C c_{2}^{\prime}$ in (5-16) and applying Lemma 2.6, we have 


$$
\begin{aligned}
& L_{1} \geq \frac{10}{|\nabla u|^{2}+2 u} \geq 2 C_{1}, \\
& L_{2} \leq c_{2}^{\prime}\left(1+\frac{r_{0}}{\sqrt{c_{2}}}+c_{3} \frac{r_{0}}{\sqrt{c_{2}}}\right), \\
& L_{3} \leq C\left(1+c_{4}\right) \frac{r_{0}^{2}}{c_{2}} .
\end{aligned}
$$

From (5-15), (5-18), and (5-17), we have

$$
\begin{aligned}
\left(1+\lambda_{1}\right) \eta & \leq \max \left\{\sqrt{\frac{2 L_{3}}{L_{1}}}, \frac{2\left(L_{2}+2\right)}{L_{1}}\right\} \\
& \leq C\left(1+\sqrt{c_{4}} \frac{r_{0}}{\sqrt{c_{2}}}+c_{2}^{\prime}\left(1+\frac{r_{0}}{\sqrt{c_{2}}}+c_{3} \frac{r_{0}}{\sqrt{c_{2}}}\right)\right) .
\end{aligned}
$$

Combining Lemma 2.6 (i) and (5-19), we have

$$
\left(1+\lambda_{1}\right)(x) \leq \frac{e^{C c_{2}^{\prime}}}{r_{0} / r_{2}-u(\tilde{x})}\left(1+\sqrt{c_{4}} \frac{r_{0}}{\sqrt{c_{2}}}+c_{2}^{\prime}\left(1+\frac{r_{0}}{\sqrt{c_{2}}}+c_{3} \frac{r_{0}}{\sqrt{c_{2}}}\right)\right)
$$

on $B\left(\tilde{x}, \frac{1}{6} \sqrt{C_{1}}\left(r_{0} / r_{2}-u(\tilde{x})\right)\right)$. The proof of Proposition 5.1 is completed.

Remark 5.2. Most computations in this section are just modifications of those in the classical theory of Monge-Ampère equations. A closer reference is [Guan and Li 1996], in which the Dirichlet problem of real Monge-Ampère equations on manifolds is systematically studied. The observation of this appendix is that these estimates can be localized under certain geometric conditions.

\section{Acknowledgements}

We are thankful to Professor J. X. Hong for beneficial comments and providing us with some references. We also thank Professor J. M. Schlenker for informing us of one interesting result in anti-de Sitter space. The first author is grateful to Professor S. T. Yau for many stimulating discussions.

\section{References}

[Cheng and Yau 1980] S. Y. Cheng and S. T. Yau, "On the existence of a complete Kähler metric on noncompact complex manifolds and the regularity of Fefferman's equation”, Comm. Pure Appl. Math. 33:4 (1980), 507-544. MR 82f:53074 Zbl 0506.53031

[Delanoë 1988] P. Delanoë, "Réalisations globalement régulières de disques strictement convexes dans les espaces d'Euclide et de Minkowski par la méthode de Weingarten", Ann. Sci. École Norm. Sup. (4) 21:4 (1988), 637-652. MR 90f:53097 Zbl 0682.53062

[Efimov 1964] N. V. Efimov, "Generation of singularities on surfaces of negative curvature", Mat. Sb. (N.S.) 64 (106) (1964), 286-320. In Russian. MR 29 \#5203 Zbl 0126.37402 
[Guan 1998] B. Guan, "The Dirichlet problem for Monge-Ampère equations in non-convex domains and spacelike hypersurfaces of constant Gauss curvature”, Trans. Amer. Math. Soc. 350:12 (1998), 4955-4971. MR 99b:53055 Zbl 0919.35046

[Guan 2007] B. Guan, "Isometric embedding of negatively curved disks in the Minkowski space", Pure Appl. Math. Q. 3:3 (2007), 827-840. MR 2008k:53067 Zbl 1154.53030

[Guan and Li 1994] P. Guan and Y. Y. Li, "The Weyl problem with nonnegative Gauss curvature", J. Differential Geom. 39:2 (1994), 331-342. MR 95c:53051 Zbl 0796.53056

[Guan and Li 1996] B. Guan and Y. Y. Li, "Monge-Ampère equations on Riemannian manifolds", J. Differential Equations 132:1 (1996), 126-139. MR 97j:53040 Zbl 0866.58067

[Guan et al. 2006] B. Guan, H.-Y. Jian, and R. M. Schoen, "Entire spacelike hypersurfaces of prescribed Gauss curvature in Minkowski space", J. Reine Angew. Math. 595 (2006), 167-188. MR 2007h:53088 Zbl 1097.53040

[Han 2005] Q. Han, "On the isometric embedding of surfaces with Gauss curvature changing sign cleanly”, Comm. Pure Appl. Math. 58:2 (2005), 285-295. MR 2005g:53106 Zbl 1073.53005

[Han 2006] Q. Han, "Local isometric embedding of surfaces with Gauss curvature changing sign stably across a curve", Calc. Var. Partial Differential Equations 25:1 (2006), 79-103. MR 2006g:53090 Zbl 1101.53001

[Han and Hong 2006] Q. Han and J.-X. Hong, Isometric embedding of Riemannian manifolds in Euclidean spaces, Mathematical Surveys and Monographs 130, Amer. Math. Soc., Providence, RI, 2006. MR 2008e:53055 Zbl 1113.53002

[Han et al. 2003] Q. Han, J.-X. Hong, and C.-S. Lin, "Local isometric embedding of surfaces with nonpositive Gaussian curvature”, J. Differential Geom. 63:3 (2003), 475-520. MR 2004i:53081 Zbl 1070.53034

[Hilbert 1901] D. Hilbert, “Ueber Flächen von constanter Gaussscher Krümmung”, Trans. Amer. Math. Soc. 2:1 (1901), 87-99. MR 1500557 JFM 32.0608.01

[Hong 1993] J. X. Hong, "Realization in $\mathbf{R}^{3}$ of complete Riemannian manifolds with negative curvature”, Comm. Anal. Geom. 1:3-4 (1993), 487-514. MR 95d:53003 Zbl 0856.53004

[Hong 1997] J. Hong, "Isometric embedding in $\mathbf{R}^{3}$ complete noncompact nonnegatively curved surfaces”, Manuscripta Math. 94:3 (1997), 271-286. MR 98m:53006 Zbl 0894.53050

[Hong 2001] J. Hong, "Recent developments of realization of surfaces in $\mathbf{R}^{3}$ ", pp. 47-62 in First International Congress of Chinese Mathematicians (Beijing, 1998), edited by L. Yang and S. T. Yau, AMS/IP Stud. Adv. Math. 20, Amer. Math. Soc., Providence, RI, 2001. MR 2002d:53081 Zbl 1056.53039

[Hong and Zuily 1995] J. Hong and C. Zuily, "Isometric embedding of the 2-sphere with nonnegative

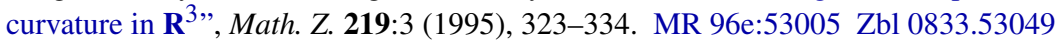

[Lin 1985] C. S. Lin, "The local isometric embedding in $\mathbf{R}^{3}$ of 2-dimensional Riemannian manifolds with nonnegative curvature", J. Differential Geom. 21:2 (1985), 213-230. MR 87m:53073 Zbl 0584.53002

[Lin 1986] C. S. Lin, "The local isometric embedding in $\mathbf{R}^{3}$ of two-dimensional Riemannian manifolds with Gaussian curvature changing sign cleanly", Comm. Pure Appl. Math. 39:6 (1986), 867-887. MR 88e:53097 Zbl 0612.53013

[Nirenberg 1953] L. Nirenberg, "The Weyl and Minkowski problems in differential geometry in the large”, Comm. Pure Appl. Math. 6 (1953), 337-394. MR 15,347b Zbl 0051.12402

[Pogorelov 1952] A. V. Pogorelov, "Regularity of a convex surface with given Gaussian curvature", Mat. Sbornik N.S. 31(73) (1952), 88-103. In Russian. MR 14,679b Zbl 0048.40501 
[Pogorelov 1973] A. V. Pogorelov, Extrinsic geometry of convex surfaces, Translations of Mathematical Monographs 35, Amer. Math. Soc., Providence, RI, 1973. MR 49 \#11439 Zbl 0311.53067

[Schlenker 2001] J.-M. Schlenker, "Surfaces à courbure extrinsèque négative dans l'espace hyperbolique", Ann. Sci. École Norm. Sup. (4) 34:1 (2001), 79-130. MR 2003d:53096 Zbl 1029.53068

[Schoen and Yau 1981] R. Schoen and S. T. Yau, "Proof of the positive mass theorem, II", Comm. Math. Phys. 79:2 (1981), 231-260. MR 83i:83045 Zbl 0494.53028

[Schulz 1990] F. Schulz, Regularity theory for quasilinear elliptic systems and Monge-Ampère equations in two dimensions, Lecture Notes in Mathematics 1445, Springer, Berlin, 1990. MR 92d:35002 Zbl 0709.35038

[Weyl 1916] H. Weyl, "Über die Bestimmung einer geschlossenen konvexen Fläche durch ihr Linienelement”, Naturf. Ges. 61 (1916), 40-72. JFM 46.1115.03

[Yau 2000] S. T. Yau, "Review of geometry and analysis", Asian J. Math. 4:1 (2000), 235-278. MR 2002e:53002 Zbl 1031.53004

Received August 28, 2014.

\section{BING-LONG CHEN}

DEPARTMENT OF MATHEMATICS

SUN YAT-SEN UNIVERSITY

GUANGZHOU, 510275

CHINA

mcscbl@mail.sysu.edu.cn

LE YIN

College of Mathematics and Computational SCIENCE

SHENZHEN UNIVERSITY

SHENZHEN, 518060

CHINA

lyin@szu.edu.cn 


\title{
PACIFIC JOURNAL OF MATHEMATICS
}

\author{
msp.org/pjm
}

Founded in 1951 by E. F. Beckenbach (1906-1982) and F. Wolf (1904-1989)

\section{EDITORS}

Don Blasius (Managing Editor)

Department of Mathematics

University of California

Los Angeles, CA 90095-1555

blasius@math.ucla.edu

\author{
Paul Balmer \\ Department of Mathematics \\ University of California \\ Los Angeles, CA 90095-1555 \\ balmer@math.ucla.edu \\ Robert Finn \\ Department of Mathematics \\ Stanford University \\ Stanford, CA 94305-2125 \\ finn@math.stanford.edu \\ Sorin Popa \\ Department of Mathematics \\ University of California \\ Los Angeles, CA 90095-1555 \\ popa@math.ucla.edu
}

\author{
Vyjayanthi Chari \\ Department of Mathematics \\ University of California \\ Riverside, CA 92521-0135 \\ chari@math.ucr.edu \\ Kefeng Liu \\ Department of Mathematics \\ University of California \\ Los Angeles, CA 90095-1555 \\ liu@math.ucla.edu \\ Jie Qing \\ Department of Mathematics \\ University of California \\ Santa Cruz, CA 95064 \\ qing@ cats.ucsc.edu
}

\section{PRODUCTION}

Silvio Levy, Scientific Editor, production@msp.org

\section{SUPPORTING INSTITUTIONS}

ACADEMIA SINICA, TAIPEI

CALIFORNIA INST. OF TECHNOLOGY

INST. DE MATEMÁTICA PURA E APLICADA

KEIO UNIVERSITY

MATH. SCIENCES RESEARCH INSTITUTE

NEW MEXICO STATE UNIV.

OREGON STATE UNIV.

\author{
STANFORD UNIVERSITY \\ UNIV. OF BRITISH COLUMBIA \\ UNIV. OF CALIFORNIA, BERKELEY \\ UNIV. OF CALIFORNIA, DAVIS \\ UNIV. OF CALIFORNIA, LOS ANGELES \\ UNIV. OF CALIFORNIA, RIVERSIDE \\ UNIV. OF CALIFORNIA, SAN DIEGO \\ UNIV. OF CALIF., SANTA BARBARA
}

\author{
Daryl Cooper \\ Department of Mathematics \\ University of California \\ Santa Barbara, CA 93106-3080 \\ cooper@math.ucsb.edu \\ Jiang-Hua Lu \\ Department of Mathematics \\ The University of Hong Kong \\ Pokfulam Rd., Hong Kong \\ jhlu@maths.hku.hk \\ Paul Yang \\ Department of Mathematics \\ Princeton University \\ Princeton NJ 08544-1000 \\ yang@math.princeton.edu
}

These supporting institutions contribute to the cost of publication of this Journal, but they are not owners or publishers and have no responsibility for its contents or policies.

See inside back cover or msp.org/pjm for submission instructions.

The subscription price for 2015 is US \$420/year for the electronic version, and \$570/year for print and electronic.

Subscriptions, requests for back issues and changes of subscribers address should be sent to Pacific Journal of Mathematics, P.O. Box 4163, Berkeley, CA 94704-0163, U.S.A. The Pacific Journal of Mathematics is indexed by Mathematical Reviews, Zentralblatt MATH, PASCAL CNRS Index, Referativnyi Zhurnal, Current Mathematical Publications and Web of Knowledge (Science Citation Index).

The Pacific Journal of Mathematics (ISSN 0030-8730) at the University of California, c/o Department of Mathematics, 798 Evans Hall \#3840, Berkeley, CA 94720-3840, is published twelve times a year. Periodical rate postage paid at Berkeley, CA 94704, and additional mailing offices. POSTMASTER: send address changes to Pacific Journal of Mathematics, P.O. Box 4163, Berkeley, CA 94704-0163.

PJM peer review and production are managed by EditFLOW ${ }^{\circledR}$ from Mathematical Sciences Publishers.

\section{PUBLISHED BY}

\section{mathematical sciences publishers \\ nonprofit scientific publishing}

http://msp.org/

(C) 2015 Mathematical Sciences Publishers 


\section{PACIFIC JOURNAL OF MATHEMATICS}

Volume $276 \quad$ No. $2 \quad$ August 2015

Free evolution on algebras with two states, II

257

MiCHAEL ANSHELEVICH

Systems of parameters and holonomicity of A-hypergeometric systems

281

Christine Berkesch Zamaere, STEPHEN GRIFfeth and EZRA Miller

Complex interpolation and twisted twisted Hilbert spaces

287

FÉlix Cabello SÁnChez, Jesús M. F. Castillo and Nigel J. Kalton

The ramification group filtrations of certain function field extensions

309

JEFFREY A. CASTAÑEDA and QINGQUAN WU

A mean field type flow, II: Existence and convergence

JEAN-BAPTISTE CASTÉRAS

Isometric embedding of negatively curved complete surfaces in Lorentz-Minkowski space

BING-LONG CHEN and LE YIN

The complex Monge-Ampère equation on some compact Hermitian manifolds

JIANCHUN CHU

Topological and physical link theory are distinct

ALEXANDER COWARD and JOEL HASS

The measures of asymmetry for coproducts of convex bodies

Qi GuO, JinFEng GuO and XunLi Su

Regularity and analyticity of solutions in a direction for elliptic equations

YongYANG Jin, DONGSHENG LI and XU-JIA WANG

On the density theorem for the subdifferential of convex functions on Hadamard spaces

Mina Movahedi, Daryoush Behmardi and Seyedehsomayeh

HOSSEINI

$L^{p}$ regularity of weighted Szegó projections on the unit disc

SAMANGi MunASINGHE and Yunus E. ZEYTUnCU

Topology of complete Finsler manifolds admitting convex functions

SORIN V. SABAU and KatSUHIRO SHIOHAMA

Variations of the telescope conjecture and Bousfield lattices for localized categories of spectra

F. LUKE WOLCOTT 